\title{
Dynamic Computation Offloading for MIMO Mobile Edge Computing Systems with Energy Harvesting
}

\author{
Wen Zhou, Ling Xing, Junjuan Xia, Lisheng Fan, and Arumugam Nallanathan, Fellow, IEEE
}

\begin{abstract}
By providing spatial diversity gain, the incorporation of multiple antennas into mobile edge computing (MEC) systems can improve the transmission performance. Meanwhile, employing energy harvesting (EH) helps enhance the system sustainability. In this paper, we focus on multi-input multi-output (MIMO) MEC systems with EH and studies the computation offloading. The design objective is to minimize the time average of a weighted sum of energy consumption and execution delay, meanwhile stabilizing the battery energy queue. To this end, we formulate the problem as a statistic program and propose a dynamic computation offloading (DCO) algorithm in which the transmitter covariance matrix, CPU-cycle frequencies for local computing, and partial offloading ratio are jointly optimized. Based on Lyapunov optimization, the program is first transformed into a nonconvex per-time slot problem. Then, we solve it by the successive convex approximation (SCA) technique, where a sequence of convex problems are created and solved. Simulation results demonstrate that the proposed algorithm is asymptotically optimal and outperforms several benchmark schemes in terms of both the average system cost and task drop ratio.
\end{abstract}

Index Terms-Mobile edge computing, MIMO, Lyapunov optimization, energy harvesting.

\section{INTRODUCTION}

Increasing mobile terminals and diversified service types bring challenges to mobile networks. To address it, mobile edge computing (MEC) is proposed to partly transfer the functions originally located in the cloud computing center to the edge of the network [1]. In this way, network congestion and system pressure can be relieved. On the other front, mobile terminals, especially small Internet of Things (IoT) devices, are often plagued by limited battery energy [2]. Fortunately, energy harvesting (EH) technology can improve this situation by capturing green energy. The integration of EH into MEC will enhance sustained computation ability of mobile devices, having potential applications such as wearable medical systems, environmental monitoring, and disaster relief, which has received a lot of attention [3]-[12].

The MEC systems with EH, termed as MEC-EH, can be classified into two types according to the EH model. For the first type, the terminal harvests radio frequency (RF) energy from the access point (AP) which usually integrates both the

W. Zhou is with the College of Information Science and Technology, Nanjing Forestry University, Nanjing 210018, China. (email: wenzhou@ustc.edu)

L. Xing is with the School of Information Engineering, Henan University of Science and Technology, Luoyang 471000, China. (email: xinglingmy@ haust.edu.cn)

J. Xia and L. Fan are both with the School of Computer Science, Guangzhou University, Guangzhou 510006, China. (email: \{xiajunjuan,lsfan\}@gzhu.edu.cn).

A. Nallanathan is with the School of Electronic Engineering and Computer Science, Queen Mary University of London, London, U.K (e-mail: a.nallanathan@qmul.ac.uk).
MEC server and energy transmitter [3]-[7]. In [3], a multiuser wireless powered MEC network was considered, where the AP broadcasted RF energy to distributed terminals. By jointly optimizing the individual mode selection and transmission time allocation, the sum computation rate was maximized. In [5], the authors studied the problem of maximizing the sum computation bits of all users in a backscatter-assisted MEC network.

While the second type of MEC-EH system adopts a more general EH model in which the terminal harvests ambient energies, including the solar energy, vibration of mechanical energy, RF energy, etc. [8]-[12]. Compared with the RF-type $\mathrm{EH}$, the ambient type is much more random, leading to that it is difficult to be predicted. The system optimization objective turns to the time average of energy consumption or execution latency. The corresponding resources scheduling policy is also parameterized by time and referred to as dynamic scheduling. In [8], the authors proposed a Lyapunov optimization-based dynamic computation offloading algorithm, to minimize the time average latency, meanwhile stabilizing the battery energy queue. In [9], the authors studied the tradeoff between the energy consumption and execution delay for MEC systems with an energy queue and several task-buffer queues.

Currently, most works on MEC networks with EH focused on the single-antenna case except that only a few works studied the multi-input single-output (MISO) case, e.g. [6]. To the best of our knowledge, there has been no work on MIMO MEC with EH. Clearly, multiple antennas can provide the spatial diversity gain and the incorporation of MIMO is able to improve the transmission performance. On the other front, the ambient energy is easily accessible and does not require an energy transmitter. Motivated by the above, we study MIMO MEC networks with the ambient-type EH and the associated offloading strategy. The contributions and novelties are summarized as follows. 1) Model: Different from the models in existing works, we incorporate MIMO into MEC networks with EH; besides, partial offloading is adopted since it is more suitable for tasks with strict latency requirement. 2) Algorithm: The system design involves both the energy consumption and execution delay and aims at minimizing the long-time average of a weighted sum of them; a dynamic computation offloading (DCO) algorithm is proposed based on successive convex approximation (SCA) [13], [14] under the Lyapunov optimization framework. 3) Results: Multiple antennas and the optimization of transmission covariance matrix help reduce the system cost and the task drop ratio.

Notations: $\mathbb{E}(\cdot)$ denotes the statistical expectation; $\mathbb{C}^{m \times n}$ represents the space consisting of $m \times n$ complex matrices. For a matrix $\mathbf{X}$, the notations $\mathbf{X}^{*}, \mathbf{X}^{H}$, and $\operatorname{Tr}(\mathbf{X})$ denote its 
conjugate, Hermitian transpose, and trace, respectively; $\mathbf{I}_{m}$ is an $m \times m$ identity matrix.

\section{System Model ANd Problem Formulation}

The system consists of an MEC server and a mobile terminal which can harvest energy from surroundings. The MEC server and terminal are equipped with $N_{T}$ and $N_{R}$ antennas, respectively. Based on the channel condition, the terminal can offload the task partially onto the MEC server. Assume that the time is slotted and the length of each slot is $\tau$. Denote the time slot set by $\Gamma \triangleq\{0,1, \cdots\}$. At the beginning of each time slot, the task of length $L$ in unit of bit arrives at the terminal with probability $\rho$, which is modelled as an i.i.d Bernoulli process. Further, assume that the task is response-time sensitive and should be finished within one time slot. Each task can be computed or simply dropped due to insufficient system resources, with an indicator $I_{\text {exe }}(n)=1(0)$ representing that the task is executed (discarded). If computed, the task can be separated into two parts: one part is computed locally while the other is offloaded and computed by the server.

\section{A. Computation Model}

At the $n$-th slot, assume that $\alpha(n) L$ bits of the task are executed locally and $(1-\alpha(n)) L$ bits are offloaded to the MEC server.

1) Local computation: The processing time for local computation is expressed as

$$
D_{l}(n)=\alpha(n) L \eta / f(n),
$$

where $\eta$ is the number of cycles of processing one bit, and $f(n)$ is the CPU frequency at time slot $n$. The energy consumption is expressed as

$$
E_{l}(n)=\alpha(n) \beta L \eta f^{2}(n),
$$

where $\beta$ is the coefficient that depends on the chip architecture.

2) MEC computation: The processing time for MEC computation is given by

$$
D_{\mathrm{MEC}}(n)=(1-\alpha(n)) L / r(\mathbf{Q}(n)),
$$

where $\mathbf{Q}(n)$ is the transmitter covariance matrix with $\operatorname{tr}(\mathbf{Q}(n)) \leq P_{T}$ and $P_{T}$ is the maximum transmission power. In (3), the uplink transmission rate is

$$
r(\mathbf{Q}(n))=B_{W} \log _{2} \operatorname{det}\left(\mathbf{I}_{N_{R}}+\mathbf{H}(n) \mathbf{Q}(n) \mathbf{H}^{H}(n) / \sigma^{2}\right),
$$

where $\mathbf{H}(n) \in \mathbb{C}^{N_{R} \times N_{T}}$ is the uplink channel matrix; $W_{B}$ is the bandwidth of the MEC sever and $\sigma^{2}$ is the noise power. Note that both the feedback delay and MEC execution delay are omitted since they are usually small. The energy consumption for the mobile terminal is given by [15]

$$
E_{\mathrm{MEC}}(n)=(1-\alpha(n)) L \operatorname{tr}(\mathbf{Q}(n)) / r(\mathbf{Q}(n)) .
$$

From (3) and (4), we can obtain an insight on the system.

3) System latency and energy consumption: With (1), (2), (3), and (4), define the total time delay and energy consumption of the system as

$$
D_{\mathrm{sys}}(n) \triangleq \mathbf{1}\left(I_{\mathrm{exe}}(n)=1\right) \cdot \max \left[D_{l}(n), D_{\mathrm{MEC}}(n)\right],
$$

$$
E_{\mathrm{sys}}(n) \triangleq \mathbf{1}\left(I_{\text {exe }}(n)=1\right) \cdot\left[E_{l}(n)+E_{\mathrm{MEC}}(n)\right],
$$

where $\mathbf{1}($.$) is the indicator function.$

\section{B. Energy Harvesting Model}

Assume that the arrived energy $E_{H}(n)$ is uniformly distributed in the interval $\left[0, E_{H}^{\mathrm{MAX}}\right]$. Clearly, the practical harvested energy $e(n)$ satisfies $0 \leq e(n) \leq E_{H}(n)$. The battery energy, denoted as $B(n)$, is updated according to the following equation

$$
B(n+1)=B(n)+e(n)-E_{\mathrm{sys}}(n) .
$$

Note that $B(n)$ refers to the energy level at the beginning of time slot $n$ [8], whereas $e(n)$ refers to the harvested energy during the period of time of length $\tau$.

For the MEC system with the RF-type EH, the collected energy at slot $n, e(n)$, can be predicted and hence it can be used for the current time slot. However, for the system with the ambient $\mathrm{EH}, e(n)$ can not be predicted and it is known at the end of slot $n$. Hence, the harvested energy $e(n)$ cannot be used at the current time slot $n$ and can only be used for the next time slot. Consequently, the system energy consumption should satisfy

$$
0 \leq E_{\mathrm{sys}}(n) \leq B(n) .
$$

\section{Problem Formulation}

The energy consumption and latency are two important factors in measuring the system performance, which are used to optimize the offloading policy. Due to the lack of battery energy or the deep fading of the channel, some tasks have to be dropped. Considering this, we introduce the task dropping cost $\Phi$ into the system cost function, which stands for a kind of 'penalization'. A large $\Phi$ means that the system prefers to execute the task, even if the channel condition is not good or the energy consumption is high, while a small $\Phi$ means the opposite. At time slot $n$, the system execution cost is defined as

$$
F_{\text {cost }}(n)=\left[E_{\mathrm{sys}}(n)+w_{D} D_{\mathrm{sys}}(n)\right]+\Phi \cdot \mathbf{1}\left(I_{\mathrm{exe}}(n)=0\right),
$$

where $w_{D}$ is the weight on the execution delay. Usually, we prefer executing a task to drop it, which leads to $\Phi \geq$ $\max \left[E_{\mathrm{sys}}(n)+w_{D} D_{\mathrm{sys}}(n)\right]$. Since $E_{\mathrm{sys}}(n)+w_{D} D_{\mathrm{sys}}(n) \leq$ $E_{\mathrm{MAX}}+w_{D} \tau$ holds, we may set $\Phi \geq E_{\mathrm{MAX}}+w_{D} \tau$.

Then, the problem is formulated as $P_{1}$. In $P_{1}, C_{1}$ is the latency constraint, $C_{2}$ and $C_{3}$ are energy constraints with $E_{\mathrm{MAx}}$ being the maximum energy consumption, $C_{5}$ is the transmission power constraint, and $C_{6}$ is the frequency constraint for local computation with $f_{\mathrm{MAX}}$ representing the maximum CPU frequency.

$$
\begin{array}{ll}
P_{1}: & \min _{\left\{I_{\text {exe }}(n), \mathbf{Q}(n), f(n), \alpha(n), e(n)\right\}} \lim _{T \rightarrow \infty} \frac{1}{T} \mathbb{E}\left[\sum_{n=0}^{T-1} F_{\text {cost }}(n)\right] \\
\text { s.t. } & C_{1}: D_{\text {sys }}(n) \leq \tau \\
& C_{2}: E_{\text {sys }}(n) \leq E_{\mathrm{MAX}} \\
& C_{3}: 0 \leq E_{\text {sys }}(n) \leq B(n) \\
& C_{4}: 0 \leq e(n) \leq E_{H}(n) \\
& C_{5}: 0 \leq \operatorname{tr}(\mathbf{Q}(n)) \leq P_{T} \cdot \mathbf{1}\left(I_{\text {exe }}(n)=1\right) \\
& C_{6}: 0 \leq f(n) \leq f_{\mathrm{MAX}} \cdot \mathbf{1}\left(I_{\text {exe }}(n)=1\right) \\
& C_{7}: 0 \leq \alpha(n) \leq 1 \\
& C_{8}: I_{\text {exe }}(n) \in\{0,1\}, n \in \Gamma .
\end{array}
$$




\section{The Proposed Dynamic Computation OFFLOADING METHOD}

In this section, we first perform transformations on $P_{1}$ to make it satisfy the prerequisite of Lyapunov optimization. Then, we solve the transformed problem.

\section{A. The DCO Algorithm}

Lyapunov optimization technique is suitable to solve the problem involving time averages and queues. $P_{1}$ is just an example of this type. However, the technique cannot be applied to it directly. Because in $C_{3}, B(n)^{\prime} s$ at different time slots are not independent so that the decision sets of $P_{1}$ at different time slots are related. This violates the prerequisite of Lyapunov optimization. To remove $C_{3}$, we introduce an energy lower bound $E_{\mathrm{MIN}}$ and construct a virtual energy queue $\tilde{B}(n)$ [8], [9].

First, introduce $E_{\mathrm{MIN}}$ into $C_{2}$, obtaining a modified version of $P_{1}$.

$$
\begin{aligned}
& P_{2}: \min _{\left\{I_{\text {exe }}(n), \mathbf{Q}(n), f(n), \alpha(n), e(n)\right\}} \lim _{T \rightarrow \infty} \frac{1}{T} \mathbb{E}\left[\sum_{n=0}^{T-1} F_{\text {cost }}(n)\right] \\
& \text { s.t. } C_{1}, C_{3}-C_{8} \\
& \quad C_{2}^{\prime}: E_{\mathrm{sys}}(n) \in\{0\} \cup\left[E_{\mathrm{MIN}}, E_{\mathrm{MAX}}\right]
\end{aligned}
$$

For $E_{\mathrm{MIN}} \rightarrow 0$, the optimal value of $P_{2}$ approaches that of $P_{1}$ [8], [9].

Second, construct a virtual energy queue $\tilde{B}(n) \triangleq B(n)-\theta$. Here, $\theta$ is a perturbation parameter satisfying

$$
\theta \geq \tilde{E}_{\mathrm{MAX}}+V\left(\Phi \cdot E_{\mathrm{MIN}}^{-1}-1\right),
$$

where $\tilde{E}_{\mathrm{MAX}}=\min \left(\beta L \eta f_{\mathrm{MAX}}^{2}+P_{T} \tau, E_{\mathrm{MAX}}\right)$ and $V$ is a control parameter. By doing so, we can ignore $C_{3}$ and the resultant solution can automatically satisfy $C_{3}$, as will be proved later.

Third, omitting $C_{3}$ of $P_{2}$, we adopt the classic Lyapunov optimization to solve it. Note that $\tilde{B}(n)$ also satisfies (7) and the queue to be stabilized in $P_{2}$ is $\tilde{B}(n)$ instead of $B(n)$. Define the Lyapunov drift function as

$$
\Delta(\tilde{B}(n))=\frac{1}{2} \mathbb{E}\left[(\tilde{B}(n+1))^{2}-(\tilde{B}(n))^{2} \mid \tilde{B}(n)\right] .
$$

Using similar technique in [8], [9], it is easy to derive that $\Delta(\tilde{B}(n)) \leq C_{0}+\mathbb{E}\left[\tilde{B}(n)\left(e(n)-E_{\text {sys }}(n)\right) \mid \tilde{B}(n)\right]$, where $C_{0}=\frac{1}{2}\left[\left(E_{H}^{\mathrm{MAX}}\right)^{2}+\tilde{E}_{\mathrm{MAX}}^{2}\right]$. Consequently, the Lyapunov drift-plus-penalty function is upper bounded by

$$
\begin{aligned}
& \Delta(\tilde{B}(n))+V\left[F_{\text {cost }}(n) \mid \tilde{B}(n)\right] \leq C_{0}+\{\tilde{B}(n) \\
& \left.\times\left[e(n)-E_{\text {sys }}(n)\right] \mid \tilde{B}(n)\right\}+V\left[F_{\text {cost }}(n) \mid \tilde{B}(n)\right] .
\end{aligned}
$$

The Lyapunov optimization-based technique minimizes the upper bound of the drift-plus-penalty function per time slot so as to reduce the system cost, meanwhile keeping the queue $\tilde{B}(n)$ stable. Based on the above, the dynamic computation offloading algorithm is proposed and summarized in Algorithm 1. Further, we have the following two lemmas about this algorithm.

Lemma 1: The optimal solution of $P_{3}$ satisfies $C_{3}$.

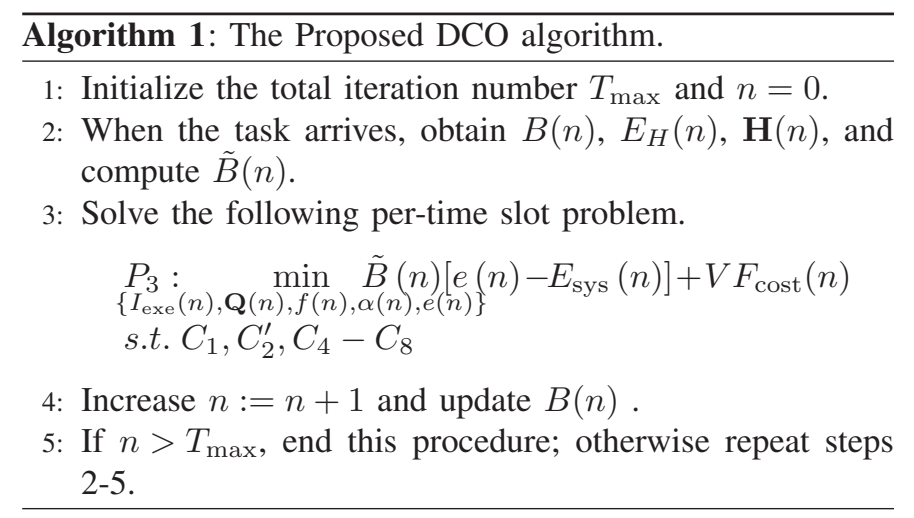

Proof: To begin with, note that the optimization of $e(n)$ only involves $C_{4}$ and is not related with other variables. The optimal $e(n)$ can be obtained by solving the sub-problem of $P_{3}$ :

$$
P_{3-1}: \min \tilde{B}(n) e(n) \text {, s.t. } C_{4} \text {. }
$$

The optimal solution is $\bar{e}(n)=E_{H}(n) \cdot \mathbf{1}(\tilde{B}(n) \leq 0)$. Then, we consider two cases. For $B(n) \geq \tilde{E}_{\mathrm{MAX}}$, it is clear that $E_{\text {sys }}(n) \leq \tilde{E}_{\mathrm{MAX}}$ and hence $E_{\text {sys }}(n) \leq B(n)$. For $B(n)<$ $\tilde{E}_{\mathrm{MAX}}$, it is clear that $\tilde{B}(n)<0$ and the objective of $P_{3}$ for executing the task is

$$
\begin{aligned}
& \tilde{B}(n)\left[E_{H}(n)-E_{\mathrm{sys}}(n)\right]+V E_{\mathrm{sys}}(n)+V w_{D} D_{\mathrm{sys}}(n) \\
& =\tilde{B}(n) E_{H}(n)+[V-\tilde{B}(n)] E_{\mathrm{sys}}(n)+V w_{D} D_{\mathrm{sys}}(n) \\
& >\tilde{B}(n) E_{H}(n)+V \Phi E_{M I N}^{-1} E_{M I N} \\
& =\tilde{B}(n) E_{H}(n)+V \Phi .
\end{aligned}
$$

Note that $\tilde{B}(n) E_{H}(n)+V \Phi$ is the objective of $P_{3}$ for dropping the task. This means that we should drop this task, which results in $E_{\mathrm{sys}}(n)=0 \leq B(n)$. Therefore, Lemma 1 holds.

Lemma 2: $\bar{F}_{\text {cost }}^{P_{2}} \leq \bar{F}_{\text {cost }}^{\mathrm{DCO}} \leq \bar{F}_{\text {cost }}^{P_{2}}+C_{0} / V$, where $\bar{F}_{\text {cost }}^{\mathrm{DCO}}$ and $\bar{F}_{\text {cost }}^{P_{2}}$ are the average costs achieved by the DCO algorithm and solving problem $P_{2}$, respectively.

The proof of Lemma 2 can be referred to [8] and [16, Section 4.5]. Here, we will not go into more details.

From the proof of Lemma 1 and Lemma 2, we can obtain the following insights on the system.

Remark 1: For $B(n)<\tilde{E}_{\mathrm{MAX}}$, the task will be dropped. This means that the system keeps on discarding tasks in order to charge the battery initially. Besides, when $\tilde{B}(n)>0$, i.e., $B(n)>\theta$, the system will discard the harvested energy. It is inferred that the battery energy is stable around the expected level $\theta$, which will be verified by simulation results later.

Remark 2: Lemma 1 implies that the solution of $P_{3}$ is also feasible for $P_{1}$ and $P_{2}$. With Lemma $2, \bar{F}_{\text {cost }}^{\mathrm{DCO}} \rightarrow \bar{F}_{\text {cost }}^{P_{2}}$ when $V \rightarrow+\infty$. Recall that $\bar{F}_{\text {cost }}^{P_{2}} \rightarrow \bar{F}_{\text {cost }}^{P_{1}}$ when $E_{\mathrm{MIN}} \rightarrow 0$. It follows that for $E_{\mathrm{MIN}} \rightarrow 0$ and $V \rightarrow+\infty, \bar{F}_{\text {cost }}^{\mathrm{DCO}} \rightarrow \bar{F}_{\text {cost }}^{P_{1}}$, where $\bar{F}_{\text {cost }}^{P_{1}}$ is the average cost of $P_{1}$. In other words, the proposed DCO method asymptotically achieves the optimal value of $P_{1}$.

\section{B. The Algorithm of Solving the Per-time Slot Problem $P_{3}$}

In the DCO algorithm, the key step is to solve the per-time slot problem $P_{3}$. This subsection will develop a SCA based 
algorithm to solve this problem. From the previous subsection, the optimal $e(n)$ is obtained by solving $P_{3-1}$ and the left is to optimize the task assignment. Since the solution for dropping the task is simple, we just consider the case of execution, which is expressed by another subproblem of $P_{3}$.

$$
\begin{aligned}
P_{3-2}: & \min _{\{\mathbf{Q}(n), f(n), \alpha(n)\}}[-\tilde{B}(n)+V] E_{\mathrm{sys}}(n)+V w_{D} D_{\mathrm{sys}}(n) \\
\text { s.t. } & C_{1}, C_{5}-C_{7} \\
& C_{2}^{\prime \prime \prime}: E_{\mathrm{MIN}} \leq E_{\mathrm{sys}}(n) \leq E_{\mathrm{MAX}}
\end{aligned}
$$

$P_{3-2}$ is non-convex due to the objective function and energy constraint. Tackling it needs the introduction of auxiliary variables and some convex transformations. This problem can be divided into two cases according to whether $-\tilde{B}(n)+V \geq 0$.

1) $-\tilde{B}(n)+V \geq 0$ : Introducing auxiliary variables $S_{1}$, $S_{2}$, and $S_{3}, P_{3-2}$ can be equivalently written as

$$
\begin{aligned}
P_{4}: & \min _{\left\{\mathbf{Q}(n), f(n), \alpha(n), S_{1}, S_{2}, S_{3}\right\}}[-\tilde{B}(n)+V]\left(S_{1}+S_{2}\right)+V w_{D} S_{3} \\
\text { s.t. } & C_{1-1}: \alpha(n) L \eta-f(n) S_{3} \leq 0 \\
& C_{1-2}:(1-\alpha(n)) L-r(\mathbf{Q}(n)) S_{3} \leq 0 \\
& C_{1-3}: S_{3} \leq \tau \\
& C_{2-1}: \beta L \eta f^{2}(n) / S_{1} \leq 1 / \alpha(n) \\
& C_{2-2}:(1-\alpha(n)) \operatorname{tr}(\mathbf{Q}(n)) / S_{2}-r(\mathbf{Q}(n)) / L \leq 0 \\
& C_{2-3}: E_{\mathrm{MIN}} \leq S_{1}+S_{2} \leq E_{\mathrm{MAX}} \\
& C_{2-4}: S_{1} \geq 0, S_{2} \geq 0 \\
& C_{5}^{\prime}: 0 \leq \operatorname{tr}(\mathbf{Q}(n)) \leq P_{T} \\
& C_{6}^{\prime}: 0 \leq f(n) \leq f_{\mathrm{MAX}} \\
& C_{7}: 0 \leq \alpha(n) \leq 1, n \in \Gamma
\end{aligned}
$$

Observe that, $C_{1-1}, C_{1-2}, C_{2-1}$ and $C_{2-2}$ are not convex and hence need convexification. Clearly,

$$
\begin{aligned}
& f_{1}(\alpha(n), f(n)) \triangleq \frac{\alpha(n)}{f(n)} \\
& \leq \frac{1}{2}\left[b_{1}{ }^{(k)}(\alpha(n))^{2}+\frac{1}{b_{1}{ }^{(k)}(f(n))^{2}}\right] \triangleq g_{1}\left(\alpha(n), f(n) ; b_{1}{ }^{(k)}\right),
\end{aligned}
$$

where $b_{1}^{(k)}$ denotes the value of $b_{1}$ in the $k$-th iteration. Using the convex upper bound of $f_{1}(\cdot)$, we replace $C_{1-1}$ by the convex constraint $C_{1-1}^{\prime}$ :

$$
\operatorname{L\eta g}_{1}\left(\alpha(n), f(n) ; b_{1}{ }^{(k)}\right) \leq S_{3} .
$$

Similarly, we have the following inequalities.

$$
\begin{aligned}
& f_{2}\left(\alpha(n), S_{3}\right) \triangleq \frac{1-\alpha(n)}{S_{3}} \\
& \leq \frac{1}{2}\left[b_{2}{ }^{(k)}(1-\alpha(n))^{2}+\frac{1}{b_{2}{ }^{(k)} S_{3}^{2}}\right] \triangleq g_{2}\left(\alpha(n), S_{3} ; b_{2}{ }^{(k)}\right), \\
& \quad f_{3}(\alpha(n)) \triangleq \frac{1}{\alpha(n)} \\
& \quad \geq \frac{1}{b_{3}^{(k)}}-\frac{1}{\left[b_{3}^{(k)}\right]^{2}}\left(\alpha(n)-b_{3}^{(k)}\right) \triangleq g_{3}\left(\alpha(n) ; b_{3}^{(k)}\right),
\end{aligned}
$$

and

$$
\begin{aligned}
& f_{4}\left(\alpha(n), \mathbf{Q}(n), S_{2}\right) \triangleq(1-\alpha(n)) \operatorname{tr}(\mathbf{Q}(n)) / S_{2} \\
& \leq \frac{1}{3}\left\{b_{4}^{(k)}(1-\alpha(n))^{3}+b_{5}^{(k)}[\operatorname{tr}(\mathbf{Q}(n))]^{3}+\frac{1}{b_{4}^{(k)} b_{5}^{(k)} S_{2}^{3}}\right\} \\
& \triangleq g_{4}\left(\alpha(n), \mathbf{Q}(n), S_{2} ; b_{4}^{(k)}, b_{5}^{(k)}\right),
\end{aligned}
$$

where $b_{2}^{(k)} \sim b_{5}^{(k)}$ have similar definitions to $b_{1}^{(k)}$. Using the inequalities above, we replace $C_{1-2}, C_{2-1}$, and $C_{2-2}$ by the following convex constraints $C_{1-2}^{\prime}, C_{2-1}^{\prime}$, and $C_{2-2}^{\prime}$, respectively.

$$
\begin{aligned}
& C_{1-2}^{\prime}: g_{2}\left(\alpha(n), S_{3} ; b_{2}{ }^{(k)}\right) \leq \frac{r(\mathbf{Q}(n))}{L} ; \\
& C_{2-1}^{\prime}: \beta L \eta f^{2}(n) / S_{1} \leq g_{3}\left(\alpha(n) ; b_{3}^{(k)}\right) \\
& C_{2-2}^{\prime}: g_{4}\left(\alpha(n), \mathbf{Q}(n), S_{2} ; b_{4}^{(k)}, b_{5}^{(k)}\right) \leq \frac{r(\mathbf{Q}(n))}{L} .
\end{aligned}
$$

With $C_{1-1}^{\prime}, C_{1-2}^{\prime}, C_{2-1}^{\prime}$ and $C_{2-2}^{\prime}$, we construct the convex problem $P_{4}^{(k)}$ that is parameterized by $b_{1}^{(k)} \sim b_{5}^{(k)}$.

$$
\begin{aligned}
& P_{4}^{(k)}: \min _{\left\{\mathbf{Q}(n), f(n), \alpha(n), S_{1}, S_{2}, S_{3}\right\}}[-\tilde{B}(n)+V]\left(S_{1}+S_{2}\right)+V w_{D} S_{3} \\
& \text { s.t. } C_{1-1}^{\prime}, C_{1-2}^{\prime}, C_{1-3}, C_{2-1}^{\prime}, C_{2-2}^{\prime}, C_{2-3}, C_{2-4}, C_{5}^{\prime}, C_{6}^{\prime}, C_{7}
\end{aligned}
$$

Note that the new constraints $C_{1-1}^{\prime}, C_{1-2}^{\prime}, C_{2-1}^{\prime}$ and $C_{2-2}^{\prime}$ are much tighter, therefore the feasible points of $P_{4}^{(k)}$ are also feasible for $P_{4}$.

Besides, when

$$
\begin{aligned}
b_{1}^{(k)} & =1 /[\alpha(n) f(n)], \\
b_{2}^{(k)} & =1 /\left[(1-\alpha(n)) S_{3}\right], \\
b_{3}^{(k)} & =\alpha(n), \\
b_{4}^{(k)} & =\operatorname{tr}(\mathbf{Q}(n)) / S_{2} /[1-\alpha(n)]^{2}, \\
b_{5}^{(k)} & =[1-\alpha(n)] / S_{2} /[\operatorname{tr}(\mathbf{Q}(n))],
\end{aligned}
$$

the functions $f_{1}(\cdot) \sim f_{4}(\cdot)$ and $g_{1}(\cdot) \sim g_{4}(\cdot)$ satisfy [14, Property A], the prerequisite of the SCA-based method.

Based on the above, the SCA-based algorithm of solving $P_{4}$ is proposed and summarized in Algorithm 2. This algorithm solves a sequence of problems $\left\{P_{4}^{(k)}\right\}$. It is easy to verify that the optimal solution of $P_{4}^{(k)}$ is feasible for $P_{4}^{(k+1)}$. Thus, the objective obtained in the $(k+1)$-th iteration is less than or equal to that in the $n$-th iteration. Since the objective, no less than zero and hence lower bounded, decreases with $n$, this algorithm is convergent. Usually, the SCA-based algorithm converges to a good local optimal solution.

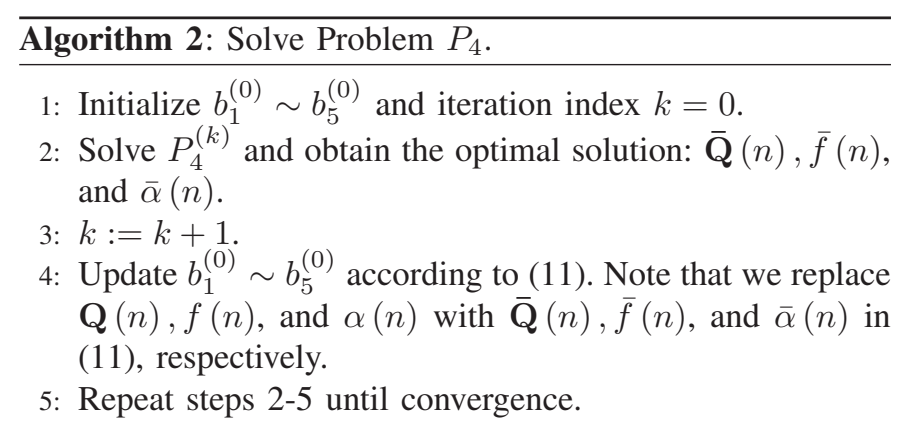

Remark 3: $b_{3}^{(k)}$ in $C_{2-1}^{\prime}$ is predetermined at the $k$-th iteration and should be treated as a constant; the function $f^{2}(n) / S_{1}$ is a quadratic-over-linear function, which is convex. Hence, $C_{2-1}^{\prime}$ is convex.

Remark 4: In $P_{4}$, the values of $S_{1} \sim S_{3}$ and the objective function depend on a sequence of $P_{4}^{(k)}$ and they can be obtained after the convergence of this sequence. From a $P_{4}^{(k)}$ 
in a single iteration, the associated convergence values cannot be obtained.

2) $-\tilde{B}(n)+V<0$ : The processing steps in this part are similar to the previous ones. Introducing auxiliary variables $S_{1}, S_{2}$, and $S_{3}$, we transform $P_{3-2}$ into an equivalent problem.

$$
\begin{aligned}
P_{5} & \min _{\left\{\mathbf{Q}(n), f(n), \alpha(n), S_{1}, S_{2}, S_{3}\right\}}[-\tilde{B}(n)+V]\left(S_{1}+S_{2}\right)+V w_{D} S_{3} \\
\text { s.t. } & C_{1-1}, C_{1-2}, C_{1-3}, C_{2-3}, C_{2-4}, C_{5}^{\prime}, C_{6}^{\prime}, C_{7} \\
& \bar{C}_{2-1}: \beta L \eta f^{2}(n) / S_{1} \geq 1 / \alpha(n) \\
& \bar{C}_{2-2}:(1-\alpha(n)) \operatorname{tr}(\mathbf{Q}(n)) / S_{2}-r(\mathbf{Q}(n)) / L \geq 0
\end{aligned}
$$

Clearly, the following inequalities hold.

$$
\begin{gathered}
f_{5}\left(S_{1}, \alpha(n)\right) \triangleq \sqrt{\frac{S_{1}}{\alpha(n)}} \leq \frac{1}{2}\left[b_{6}^{(k)} S_{1}+\frac{1}{b_{6}^{(k)} \alpha(n)}\right] \\
\triangleq g_{5}\left(S_{1}, \alpha(n) ; b_{6}^{(k)}\right), \\
f_{6}\left(S_{2}, \alpha(n), \mathbf{Q}(n)\right) \triangleq \frac{S_{2}}{(1-\alpha(n) \operatorname{tr}(\mathbf{Q}(n))} \leq \frac{1}{3}\left\{b_{7}^{(k)} S_{2}^{3}+\right. \\
\left.\frac{b_{8}^{(k)}}{[1-\alpha(n)]^{3}}+\frac{\left[b_{7}^{(k)} b_{8}^{(k)}\right]^{-1}}{[\operatorname{tr}(\mathbf{Q}(n))]^{3}}\right\} \triangleq g_{6}\left(S_{2}, \alpha(n), \mathbf{Q}(n) ; b_{7}^{(k)}, b_{8}^{(k)}\right),
\end{gathered}
$$

and

$$
\begin{aligned}
& f_{7}(\mathbf{Q}(n)) \triangleq \frac{1}{r(\mathbf{Q}(n))} \geq r\left(\mathbf{Q}_{0}^{(k)}\right)-\frac{1}{\left[r\left(\mathbf{Q}_{0}^{(k)}\right)\right]^{2}} \times \\
& \operatorname{tr}\left[\nabla_{\mathbf{Q}^{*}(n)} r\left(\mathbf{Q}_{0}^{(k)}\right)\left(\mathbf{Q}(n)-\mathbf{Q}_{0}^{(k)}\right)\right] \triangleq g_{7}\left(\mathbf{Q}(n) ; \mathbf{Q}_{0}^{(k)}\right),
\end{aligned}
$$

where $\nabla_{\mathbf{Q}^{*}(n)} r(\cdot)$ is the gradient of $r(\cdot)$ with respect to the conjugate of $\mathbf{Q}(n)$ and

$\nabla_{\mathbf{Q}^{*}(n)} r\left(\mathbf{Q}_{0}^{(k)}\right)=\frac{B_{W} \mathbf{H}^{H}(n)}{\ln 2 \cdot \sigma^{2}}\left[\mathbf{I}+\frac{\mathbf{H}(n) \mathbf{Q}_{0}^{(k)} \mathbf{H}^{H}(n)}{\sigma^{2}}\right]^{-1} \mathbf{H}(n)$.

Note that, since both $\nabla_{\mathbf{Q}^{*}(n)} r(\cdot)$ and $\left(\mathbf{Q}(n)-\mathbf{Q}_{0}^{(k)}\right)$ are Hermitian, it is easy to verify that $\operatorname{tr}\left[\nabla_{\mathbf{Q}^{*}(n)} r\left(\mathbf{Q}_{0}^{(k)}\right)\left(\mathbf{Q}(n)-\mathbf{Q}_{0}^{(k)}\right)\right]$ is a real number.

We use the above inequalities to convexify $\bar{C}_{2-1}$ and $\bar{C}_{2-2}$, obtaining

$$
\begin{aligned}
& \bar{C}_{2-1}^{\prime}: \sqrt{\beta L \eta} f(n) \leq g_{5}\left(S_{1}, \alpha(n) ; b_{6}^{(k)}\right) \\
& \bar{C}_{2-2}^{\prime}: g_{6}\left(S_{2}, \alpha(n), \mathbf{Q}(n) ; b_{7}^{(k)}, b_{8}^{(k)}\right) \geq L \cdot g_{7}\left(\mathbf{Q}(n) ; \mathbf{Q}_{0}^{(k)}\right) .
\end{aligned}
$$

With $C_{1-1}^{\prime}, C_{1-2}^{\prime}, \bar{C}_{2-1}^{\prime}$ and $\bar{C}_{2-2}^{\prime}$, construct the convex problem $P_{5}^{(k)}$, parameterized by $b_{1}^{(k)}, b_{2}^{(k)}, b_{6}^{(k)} \sim b_{8}^{(k)}$, and $\mathbf{Q}_{0}^{(k)}$.

$$
\begin{aligned}
& P_{5}^{(k)}: \min _{\left\{\mathbf{Q}(n), f(n), \alpha(n), S_{1}, S_{2}, S_{3}\right\}}[-\tilde{B}(n)+V]\left(S_{1}+S_{2}\right)+V w_{D} S_{3} \\
& \text { s.t. } C_{1-1}^{\prime}, C_{1-2}^{\prime}, C_{1-3}, \bar{C}_{2-1}^{\prime}, \bar{C}_{2-2}^{\prime}, C_{2-3}, C_{2-4}, C_{5}^{\prime}, C_{6}^{\prime}, C_{7}
\end{aligned}
$$

Besides, when (11a)-(11b) hold and

$$
\begin{aligned}
& b_{6}^{(k)}=1 / \sqrt{S_{1} \alpha(n)}, \\
& b_{7}^{(k)}=1 /\left[(1-\alpha(n)) \operatorname{tr}(\mathbf{Q}(n)) S_{2}^{2}\right], \\
& b_{8}^{(k)}=S_{2}(1-\alpha(n))^{2} / \operatorname{tr}(\mathbf{Q}(n)), \\
& \mathbf{Q}_{0}^{(k)}=\mathbf{Q}(n),
\end{aligned}
$$

the functions $f_{5}(\cdot) \sim f_{7}($.$) and g_{5}(\cdot) \sim g_{7}($.$) satisfy [14,$ Property A].

Based on the above, it is easy to design the algorithm to solve $P_{5}$. Since it is similar to Algorithm 2 in structure, we omit the details. The main differences from Algorithm 2 lie in two aspects: 1) Step 1 initializes $b_{1}^{(0)}, b_{2}^{(0)}, b_{6}^{(0)} \sim b_{8}^{(0)}, \mathbf{Q}_{0}^{(k)}$ instead of $b_{1}^{(0)} \sim b_{5}^{(0)}$;2) Step 4 updates these variables according to (11a), (11b), and (12). Finally, we present the algorithm of solving the per-time slot problem $P_{3}$, summarized in Algorithm 3.

Algorithm 3 : SCA-based Algorithm for the Per-time Slot Problem $P_{3}$.

1: Obtain the optimal harvested energy $\bar{e}(n)=E_{H}(n)$. $\mathbf{1}(\tilde{B}(n) \leq 0)$.

2: If $-\tilde{B}(n)+V \geq 0$, solve $P_{4}$ by Algorithm 2; otherwise, solve $P_{5}$ by the similar algorithm. Denote the optimal solution and its objective as $\{\overline{\mathbf{Q}}(n), \bar{f}(n), \bar{\alpha}(n)\}$ and $\bar{F}_{\text {obj }}$, respectively.

3: If $\bar{F}_{\text {obj }}<V \Phi$, set the optimal $\bar{I}_{\text {exe }}(n)=1$ and output $\left\{\bar{I}_{\text {exe }}(n), \overline{\mathbf{Q}}(n), \bar{f}(n), \bar{\alpha}(n), \bar{e}(n)\right\} ;$ otherwise $\bar{I}_{\text {exe }}(n)=0$ and output $\left\{\bar{I}_{\text {exe }}(n), \bar{e}(n)\right\}$.

Remark 5: The problem $P_{4}$ can be solved by the block coordinate descent (BCD) approach, by which the coupling among variables of $P_{4}$ can be tackled. However, it can not solve $P_{5}$ - another subproblem of the per-time slot problem $P_{3}$, where the main obstacle lies in the constraint $\bar{C}_{2-2}$. Because when optimizing $\mathbf{Q}(n)$, the constraint $\bar{C}_{2-2}$ is not convex even if we fix all other variables. In summary, the BCD approach can solve $P_{4}$ but not $P_{5}$, leading to that it cannot solve $P_{3}$. To give a uniform framework for the solution of problems $P_{3}, P_{4}$ and $P_{5}$, we adopt the SCA-based algorithm in this work.

\section{Simulation Results}

In this section, computer simulation is deployed to investigate the performance of the proposed algorithm. The uplink channel is modeled as $\mathbf{H}(n)=\frac{0.01}{d^{2}} \mathbf{H}_{\omega}(n)$, where $d$ is the distance between transmitter and receiver and set to $80 \mathrm{~m}$; $\mathbf{H}_{\omega}(n)$ is a random matrix with i.i.d. zero-mean and unit variance complex Gaussian random variables. For the mobile terminal, the task of length $L=2000$ bits arrives with probability $\rho=0.4$; the arrived energy is uniformly distributed in $0 \sim E_{H}^{\mathrm{MAX}}=120 \mu \mathrm{J}$. Besides, $\beta=10^{-28} \mathrm{~J} \cdot \mathrm{s}^{2}$, $\tau=2 \mathrm{~ms}, E_{\mathrm{MAX}}=1 \mathrm{~mJ}, \Phi=2 \mathrm{~mJ}, B_{W}=1 \mathrm{MHz}$, $P_{T}=1 \mathrm{~W}, f_{\mathrm{MAX}}=1.5 \mathrm{GHz}, \eta=750 \mathrm{cycles} / \mathrm{bit}$, the noise power $\sigma^{2}=3.4 \times 10^{-13} \mathrm{~W}$. Unless otherwise specified, $N_{T}=N_{R}=2$, the weight factors $V=0.16 \mathrm{~mJ}$ and $w_{D}=0.5 \mathrm{~W}, E_{\mathrm{MIN}}=0.05 \mathrm{~mJ}$, and the expected battery level $\theta=\tilde{E}_{\mathrm{MAX}}+V\left(\Phi \cdot E_{\mathrm{MIN}}^{-1}-1\right)$.

Five benchmark schemes are included for comparison: the local-only scheme [9], MEC-only scheme [9], greedy offloading scheme [9], isotropic transmission, and the 'DCO-f' scheme. The isotropic transmission means the proposed DCO with fixed $\mathbf{Q}(n)=\sqrt{P_{T}} \mathbf{I}$; the 'DCO-f' scheme refers to the proposed DCO with fixed $f(n)=f_{\text {MAX }}$.

Observe from Fig. 1(a) that the battery energy of the proposed DCO increases in the first $0.4 \mathrm{~s}$ and keeps stable 
around the expected battery level. For the other two schemes, their energies fluctuate at a lower level due to lack of effective energy management. In Fig. 1 (b), the average cost of DCO converges to $0.2 \mathrm{~mJ}$, lower than the costs achieved by the localonly and greedy offloading schemes. However, its convergence speed is slow because the battery energy management forces many tasks to be abandoned at the beginning, resulting in a high initial cost. In Fig. 2, with increasing $V$ and decreasing $E_{\mathrm{MIN}}$, DCO's cost decreases and it converges to around 0.2 $\mathrm{mJ}$ eventually, which verifies DCO's asymptotic optimality mentioned in Lemma 2. Given appropriate $V$ and $E_{\mathrm{MIN}}$, e.g., $V \geq 0.1$ and $E_{\mathrm{MIN}} \leq 0.1$, the proposed DCO is able to achieve the lowest average cost among the six schemes.

Table I presents the task drop ratios under six schemes. When the number of antennas increases, resulting in more channel gains, the drop ratios for the proposed DCO, istropic, DCO-f, MEC-only and Greedy offloading decrease. Clearly, the drop ratio of the DCO is always the lowest among the six schemes.

To sum up, the proposed DCO is superior to other schemes in terms of the average cost and drop ratio performance.

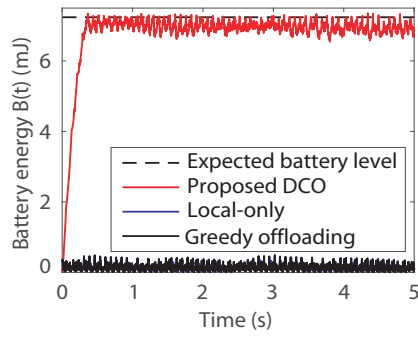

(a)

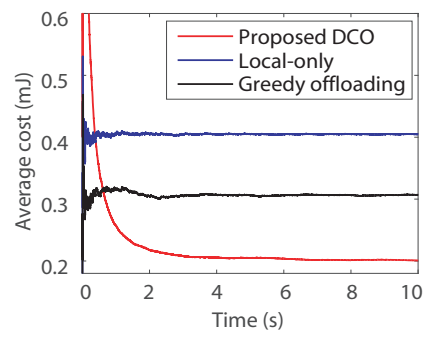

(b)
Fig. 1 Battery energies and average costs of several schemes v.s. time.

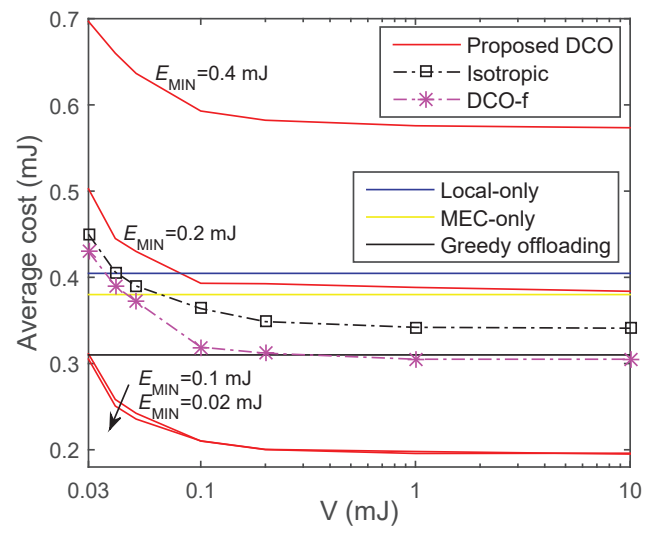

Fig. 2 Average cost v.s. $V$.

TABLE I

TASK DROP RATIOS OF DIFFERENT SCHEMES

\begin{tabular}{|c|c|c|c|}
\hline \multirow{2}{*}{ Schemes } & \multicolumn{3}{|c|}{ Number of antennas $\left(N_{T}=N_{R}\right)$} \\
\cline { 2 - 4 } & 1 & 2 & 3 \\
\hline DCO & 0.0020 & 0 & 0 \\
\hline Local-only & 0.090 & 0.090 & 0.090 \\
\hline MEC-only & 0.66 & 0.26 & 0.043 \\
\hline Greedy & 0.088 & 0.061 & 0.0075 \\
\hline Isotropic & 0.0040 & 0 & 0 \\
\hline DCO-f & 0.26 & 0.15 & 0 \\
\hline
\end{tabular}

\section{CONCLusions}

In this letter, we proposed a DCO scheme for MIMO MEC-EH systems, where the offloading ratio, transmission covariance matrix, and CPU-cycle frequency were jointly optimized to minimize the average weighted sum of energy consumption and latency. Further, two lemmas were presented to support the proposed scheme. Simulation results show that, given appropriate parameters, the proposed DCO algorithm has better performance than the benchmark schemes, in terms of both the system cost and task drop ratio. For the future work, we would like to extend the findings in this work to the scenarios with multiple edge users or with complicated noise [17].

\section{REFERENCES}

[1] C. Li, "Dynamic offloading for multiuser muti-CAP MEC networks: A deep reinforcement learning approach," IEEE Trans. Veh. Technol., vol. PP, pp. 1-5, 2021.

[2] G. Gui, M. Liu, F. Tang, N. Kato, and F. Adachi, "6G: Opening new horizons for integration of comfort, security and intelligence," IEEE Wireless Commun., doi: 10.1109/MWC.001.1900516.

[3] S. Bi and Y. J. Zhang, "Computation rate maximization for wireless powered mobile-edge computing with binary computation offloading," IEEE Trans. Wireless Commun., vol. 17, no. 6, pp. 4177-4190, 2018.

[4] Q. Gu, Y. Jian, G. Wang, R. Fan, H. Jiang, and Z. Zhong, "Mobile edge computing via wireless power transfer over multiple fading blocks: An optimal stopping approach," IEEE Trans. Veh. Technol., vol. 69, no. 9, pp. 10348-10361, 2020.

[5] L. Shi, Y. Ye, X. Chu, and G. Lu, "Computation bits maximization in a backscatter assisted wirelessly powered MEC network," IEEE Commun. Lett., 2020, doi: 10.1109/LCOMM.2020.3027294.

[6] F. Wang, J. Xu, X. Wang, and S. Cui, "Joint offloading and computing optimization in wireless powered mobile-edge computing systems," IEEE Trans. Wireless Commun., vol. 17, no. 3, pp. 1784-1797, 2018.

[7] F. Wang, J. Xu, and S. Cui, "Optimal energy allocation and task offloading policy for wireless powered mobile edge computing systems," IEEE Trans. Wireless Commun., vol. 19, no. 4, pp. 2443-2459, 2020.

[8] Y. Mao, J. Zhang, and K. B. Letaief, "Dynamic computation offloading for mobile-edge computing with energy harvesting devices," IEEE $J$. Sel. Areas Commun., vol. 34, no. 12, pp. 3590-3605, 2016.

[9] G. Zhang, W. Zhang, Y. Cao, D. Li, and L. Wang, "Energy-delay tradeoff for dynamic offloading in mobile-edge computing system with energy harvesting devices," IEEE Trans. Ind. Informat., vol. 14, no. 10, pp. 4642-4655, 2018.

[10] M. Merluzzi, P. D. Lorenzo, and S. Barbarossa, "Latency-constrained dynamic computation offloading with energy harvesting IoT devices," in Proc. IEEE INFOCOM, Paris, France, 2019, pp. 750-755.

[11] Y. Deng, Z. Chen, X. Yao, S. Hassan, and A. M. A. Ibrahim, "Parallel offloading in green and sustainable mobile edge computing for delayconstrained IoT system," IEEE Trans. Veh. Technol., vol. 68, no. 12, pp. 12 202-12 214, 2019.

[12] Z. Wei, B. Z. andJ. Su, and X. Lu, "Dynamic edge computation offloading for internet of things with energy harvesting: A learning method," IEEE Internet Things J., vol. 6, no. 3, pp. 4436-4447, 2019.

[13] G. Scutari, F. Facchinei, and L. Lampariello, "Parallel and distributed methods for constrained nonconvex optimization-Part I: Theory," IEEE Trans. Signal Process., vol. 65, no. 8, pp. 1929-1944, 2017.

[14] A. Beck, A. Ben-Tal, and L. Tetruashvili, "A sequential parametric convex approximation method with applications to nonconvex truss topology design problems," J. Global Optim., vol. 47, no. 1, pp. 29$51,2010$.

[15] S. Sardellitti, G. Scutari, and S. Barbarossa, "Joint optimization of radio and computational resources for multicell mobile-edge computing," IEEE Trans. Signal Inf. Process. Netw., vol. 1, no. 2, pp. 89-103, 2014.

[16] M. J. Neely, Stochastic Network Optimization With Application to Communication Queueing Systems. San Rafael, CA, USA: Morgan and Calypool, 2010.

[17] K. He, "Learning based signal detection for MIMO systems with unknown noise statistics," IEEE Trans. Commun., vol. PP, pp. 1-10, 2021. 